\title{
Performance Analysis of Power Consumption in LTE-A eNB and its Effect on the Overall System Efficiency
}

\author{
Eric Sackey, Rajeev Paulus \\ Department of Electronics and Communications Engineering, Higgingbottom of Agriculture, Technology and Sciences,
} (SHUATS), Allahabad, Uttar Pradesh, India

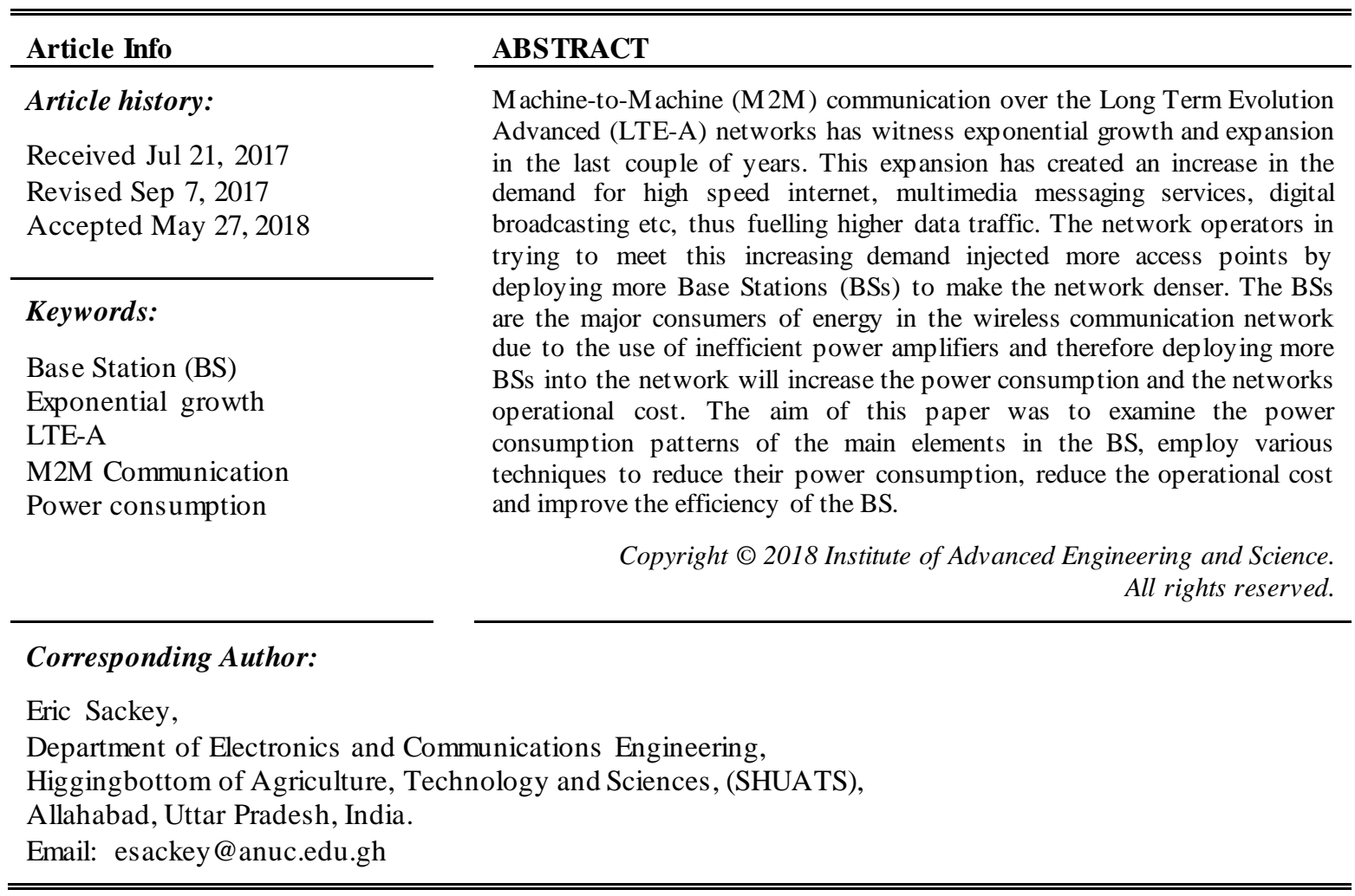

\section{INTRODUCTION}

Machine-to-Machine (M2M) communication has seen an astronomical growth and expansion in the last couple of years. This growth and expansion is the direct result of the rapid growth in the number of personal computers, smart-phones, tablets just to mention a few. This growth has escalated the demand for high speed internet connectivity, online gaming, multi-media messaging services, and digital broadcasting thus fuelling higher data traffic flowing into the network. M2M communication is the latest addition to the wireless communication system. M2M communication is a network of connected devices or objects with amazing intelligence and sensing capabilities often designed to perform certain tasks with little or no human interventions. M2M communication has permeated every sphere of human endeavour with it unlimited range of applications. As a matter of fact it is perceived as the main driving force behind the future internet of things (IoT). The LTE/4G networks are considered to be the most viable infrastructural option in the speedy and successful implementation of M2M communication in the immediate future [1].

The LTE and LTE-A technologies primarily use OFDM as their basis with the objectives to improve system capacity, low latency, enhanced network coverage, reduce operating cost, bandwidth operational flexibility and smooth integration of the existing systems [2]. This leads to improving the spectral efficiency per unit area of the existing system, and can be achieved with the deployment of Heterogeneous Networks (HetNet) involving microcell, picocell, femtocell and relay nodes of low power underneath the macrocell Based Station also known eNB. Deployment of these BS underneath the macrocell will improve the spectral efficiency per unit area and bring about enhanced user experience within the network [3]. Injecting these BSs into the network increases the access points thus creating a denser network [4]. Power Efficiency (PE) is 
becoming an area of great concern owing to the growing awareness of the global Carb on Dioxide $(\mathrm{CO} 2)$ emission [5]. It has been observed that the ICT sector presently is responsible for $4-6 \%$ of carbon emission globally and considering the rate at which wireless communication is growing and expanding if pragmatic steps are not taken to curb it, these numbers will certainly swell up further [6]. Again the eNBs are the net consumers of energy in the wireless communication network [7, 8] so increasing the number of network access points by deploying more eNBs will mean the amount of energy con sumed in the network increasing accordingly and hence the volume of carbon emission into the atmosphere [9-11]. The eNBs are the net consumers of energy in the wireless communication networks owing to their continual usage of inefficient power amplifiers (PA) $[12,13]$. The researchers aim in this paper was to examine the power consumption patterns of the main elements of the eNBs with the view of identifying various techniques to improve the power efficiency of the BSs which will go a long way in improving the network efficiency as a whole. The contribution of this work is that the researchers explored the use of more power efficient amplifiers (Doherty power amplifier) [13, 14], incorporated load switching mode into the operation of the eNBs and employed Multiple Input Multiple Output (MIMO) into the eNBs antennas configuration to overcome the aforementioned problem under various load conditions.

The rest of the paper is organized as follows: Section 2 provides a review of some related literature while description of the LTE/LTEA system model of the eNB elements and the formulation problem is presented in Section 3. Section 4 presents the analysis of the simulation results. Finally, section 5 concludes the paper.

\section{LITERATURE REVIEW}

Large volume of literature exists on how to improve the efficiency of the eNBs. For instance, the authors in [15] evaluated how the energy efficiency of the eNB can be improved by utilizing the sleep mode in a dense LTE network. In [16] the authors proposed resource block allocation and transmit power control to improve the energy efficiency in the down link of the LTE system. The author in [17] demonstrated that the output power requirement of the microcell eNB can be improved by as much as $65.3 \%$ by employing the Doherty power amplifier as against the conventional power amplifier. The authors [18] also showed that by using class F Doherty power amplifier a high efficiency can be obtained for LTE-A application due to the suppression of the second and third order harmonic component and thereby obtaining a linear out put. The authors in $[19,20]$ did an extensive work on the energy efficiency by comparing the bit rate of the various eNBs with the amount of power consumed by these eNBs. The novelty in this work lies in the fact that the comparison is made between the power consumed by the macrocell eNBs and the microcell eNBs based on the number of active users when these eNBs are operating under (a) single or multiple transmitting antennas, (b) doherty or conventional power amplifiers and (c) proposed load switching when the eNB is operating under no-load condition.

\subsection{System Model}

One of the objectives of the LTE- release 13 is to reduce the operational cost of the LTE-A networks. In this regards the researchers will examine the power consumption pattern of the key elements in the eNBs with the view of optimizing their operations to improve the overall network efficiency. In this work, heterogeneous networks involving macrocell and microcell were used to improve the user experience within the network. The researchers evaluated the power efficiency of a microcell as against the macrocell. The main elements of macrocell eNBs are:

1. Rectifier $-100 \mathrm{~W}$ used for converting the incoming alternating current to direct current, as shown in Table 1.

2. Digital Signal Processing (DSP) unit $-100 \mathrm{~W}$ involves the conversion of audio and other analog signal into digital format such as bits that can be processed and vice versa.

3. Transceiver $1000 \mathrm{~W}$ for the transmission and reception of signals.

4. Power Amplifier (PA) $-1000 \mathrm{~W}$ responsible for changing the input dc power into a signal strong enough to drive a radio frequency $(\mathrm{RF})$ signal.

5. Microwave or Fibre Link $80 \mathrm{~W}$ used to link the transmitting antenna with the transceiver unit.

6. Air condition and lighting $-220 \mathrm{~W}$ used to control the ambient temperature within the BS compartment and to provide lighting at the eNBs, as shown in Figure 1 [21]. 
Table 1. Power pattern of the key elements at the macrocell and microcell base stations

\begin{tabular}{ccc}
\hline BS Elements & $\begin{array}{c}\text { Macrocell Base Station } \\
\text { power }(\mathrm{W})\end{array}$ & $\begin{array}{c}\text { Microcell Base Station } \\
\text { power }(\mathrm{W})\end{array}$ \\
\hline Power Amplifier & 1000 & 100 \\
Transceiver & 1000 & 100 \\
Digital Signal Processing & 100 & 80 \\
Rectifier & 100 & 60 \\
Air condition & 220 & 100 \\
Microwave or fibre link & 80 & - \\
\hline
\end{tabular}

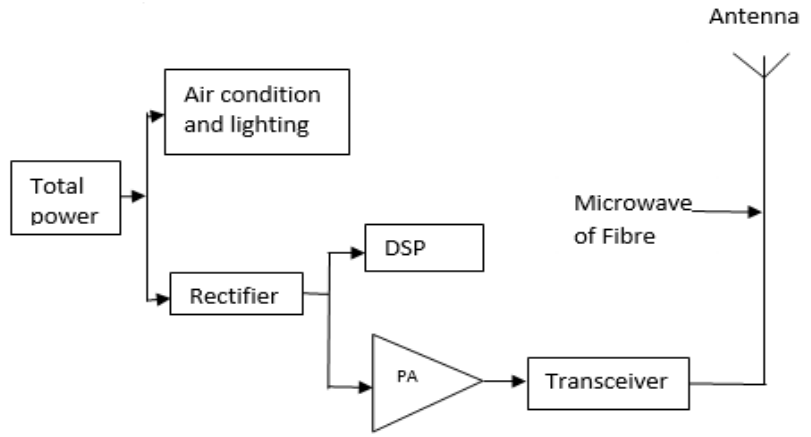

Figure 1. System model of Macrocell eNB Base Station [5, 14]

\subsection{Formulation of problem}

When these elements are combined one can find the sum total of the power consumed at the eNBs, however some of the elements are used in multiple times, take for instance the antennas. Since use is made $120^{\circ}$ sector antennas means it is required to use three antennas to cover a given area, which also implies the use three of PA, DSP, Transceiver and Rectifier each. The amount of power consumed by the microwave link and rectifier remains constant. The amount of power consumed by air condition is determined by the temperature within and outside the eNBs compartment. The power consumption pattern of Digital Signal Processing unit, Power Amplifier and the transceiver is influenced by the load factor $(F)$ which represents a fraction of the number of active users on eNBs. This load factor $(F)$ may vary from zero to one $(0-1)$ depending what time of the day and the location of the eNBs [22]. Take for instance eNBs located at event centres such as stadia or shopping malls, unless occasions or there is an event taking place at such places the full capacity of the eNBs will not be fully utilised. Installing an eNB at the central business centre means that at night when most businesses have closed the eNBs will be underutilised [23]. In such instances operating the eNBs at its full installed capacity will amount to wasting the power and resources of the eNBs for which there will be no gain in return. In this case part of the eNBs must be switched off during off-load periods based on the eNBs configuration to conserve energy. The management operations of turning off part of the eNB lead to the development of Self Organizing Networks (SON) [9]. The network operator has to switch off part the eNB in order the save energy when the eNB carries no traffic [24]. SON was developed to enable the network operators to automatically switch off any eNB which is underutilised so as to save the network operators of any unnecessary operational cost during off-load (no-load) periods while making a significant power saving or reduction [25], [26]. The LTE-A system should be flexible enough to ensure that M2M devices serving as data originators (utility meters and sensors) can remain in the power saving mode for longer period and be able to readily reconnect to the network in its next data genera ting cycle to prolong their battery life span [27], [28]. The total power consumed by the macrocell eNBs is given as:

$$
\mathrm{P}_{\text {Total }_{(\text {Macro })}}=\mathrm{n}_{\text {Sec }}\left(\mathrm{P}_{\text {Rect }}+\mathrm{F} \times\left(\mathrm{P}_{\text {Amp }}+\mathrm{P}_{\text {Trans }}+\mathrm{P}_{\mathrm{DSP}}\right)\right)+\mathrm{P}_{\text {Link }}+\mathrm{P}_{\text {Aircon }}
$$

Where

$$
\mathrm{P}_{\mathrm{Amp}}=\frac{\mathrm{P}_{\mathrm{Trans}}}{\eta_{\mathrm{PA}}}
$$

and $\eta_{\mathrm{PA}}$ is the efficiency of the power amplifier. 
The main elements of the microcell BS are:

1. Rectifier $60 \mathrm{~W}$

2. Digital Signal Processing unit $80 \mathrm{~W}$

3. Power Amplifier (PA) $100 \mathrm{~W}$

4. Transceiver $100 \mathrm{~W}$

5. Air condition $100 \mathrm{~W}$

All the elements used in the microcell BS are the same as those used in the macrocell eNBs with the exception of the omni-directional antenna and here there is no microwave link in the microcell BS [29]. The total power consumed by the microcell BS is given as

$$
\begin{aligned}
& \mathrm{P}_{\text {Total }_{(\text {Micro }}}=\mathrm{P}_{\text {Rect }}+\mathrm{F} \times\left(\mathrm{P}_{\text {Amp }}+\mathrm{P}_{\text {Trans }}+\mathrm{P}_{\mathrm{DSP}}\right)+\mathrm{P}_{\text {Aircon }} \\
& \mathrm{P}_{\text {Amp }}=\frac{\mathrm{P}_{\text {Trans }}}{\eta_{\mathrm{PA}}}
\end{aligned}
$$

where

1. $P_{\text {Rect }}-$ the amount of power used for rectification at the BS.

2. $\mathrm{P}_{\mathrm{Amp}}-$ the amount of power consumed by the power amplifier.

3. $\mathrm{P}_{\text {Trans }}$ - amount of power consumed by the transceiver.

4. $\mathrm{P}_{\mathrm{DSP}}-$ the amount of power consumed by the digital signal processing unit.

5. $\mathrm{P}_{\text {Link }}$-the amount of power consumed by the microwave or fibre link.

6. $\mathrm{P}_{\text {Aircon }}$-the amount of power consumed by air condition and lighting points

$\eta_{\mathrm{PA}}$ is the efficiency of the power amplifier. The performance efficiency of the conventional power amplifier ranges from 15-20 percent, in this case selection is based on best performance of 20 percent [24], [30]. To improve the performance of the eNBs however an efficient power amplifier is introduced as Doherty Power Amplifier with efficiency as high in the range of 50-65 percent [18], [19]. In this case best performance of 65 percent was selected.

\section{PERFORMANCE ANALYSIS OF SNR, BER AND TRANSMITTED POWER IN LTE-A WIRELESS COMMUNICATION BETWEEN SINGLE ANTENNA AND MULTIPLE ANTENNA SYSTEMS}

a) Wireless Communication System Model

$$
y=h x+n
$$

where $\mathrm{y}=$ received signal, $\mathrm{h}=$ fading channel coefficient, $\mathrm{x}=$ transmitted signal and $\mathrm{n}=$ Additive White Gaussian Noise (AWGN) respectively.

The received power

$$
P_{r}=|h|^{2} x^{2}=|h|^{2} P=a^{2} P
$$

The performance of the wireless communication system is degraded when the received power is less than the noise power.

$$
\begin{aligned}
& a^{2} P<{\sigma_{n}}^{2} \\
& a^{2}<\frac{\sigma_{n}{ }^{2}}{P}
\end{aligned}
$$

But signal to noise ratio (SNR) is given as:

$$
\begin{aligned}
& S N R=\frac{P}{\sigma_{n}^{2}} \\
& a=\frac{1}{\sqrt{S N R}}
\end{aligned}
$$

When $a<1 / \sqrt{S N R}$, the received power is less than the noise power at the receiver, this means that the bits error rate (BER) is going to be very high and this phenomenon is known as Deep Fade. 
b) Probability Density Function of a Rayleigh Fading Channel

$$
\int A(a)=2 a e^{-a^{2}}
$$

The probability of a deep fade event

$$
\begin{aligned}
p\left(a<\frac{1}{\sqrt{S N R}}\right) & =\int_{0}^{1 / \sqrt{S N R}} f A(a) d a \\
& =\int_{0}^{1 / \sqrt{S N R}} 2 a e^{-a^{2}} d a
\end{aligned}
$$

At high SNR the whole equation can be approximated as:

$$
\begin{aligned}
p\left(a<\frac{1}{\sqrt{S N R}}\right) & =\int_{0}^{1 / \sqrt{S N R}} 2 a d a \\
& =\left.\frac{2 a^{2}}{2}\right|_{0} ^{\frac{1}{\sqrt{S N R}}} \\
& =\frac{1}{S N R}
\end{aligned}
$$

The probability of deep fade in a wireless communication system is $\frac{1}{S N R}$

But the BER is $\frac{1}{2 S N R}$

\section{c) Received Antenna Diversity}

One of the objectives of LTE-Advanced systems is to provide ubiquitous wireless network. Deep fading degrades the performance of the wireless network is therefore undesired in LTE-A system. Antenna Diversity is one method that can be used to overcome deep fade effect in LTE-Advanced.
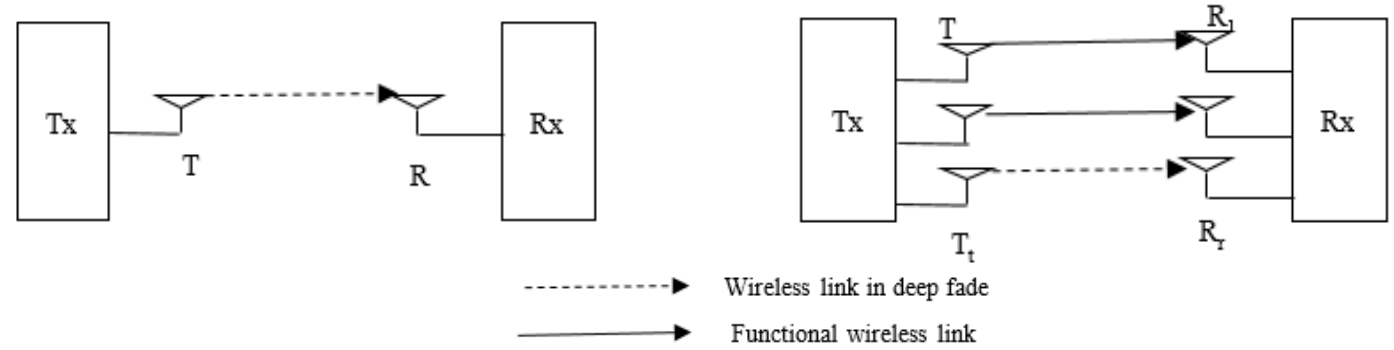

(a). Single link wireless link

(b). Wireless system with diversity

Figure 2. Diagram of wireless system with antenna diversity

Let the received signal be

$$
y=h x+n
$$

and $\mathrm{x}$ be the trans mitted signal.

$$
y_{1}=h_{1} x+n_{1}
$$




$$
\begin{gathered}
y_{2}=h_{2} x+n_{2} \\
\vdots \\
y_{L}=h_{L} x+n_{L}
\end{gathered}
$$

Where $h_{1}, h_{2}, \quad h_{L}$ are the fading coefficients of links.

\section{1) System Model}

$$
\left[\begin{array}{c}
\mathrm{y}_{1} \\
\mathrm{y}_{2} \\
\vdots \\
\mathrm{y}_{\mathrm{L}}
\end{array}\right]=\left[\begin{array}{c}
\mathrm{h}_{1} \\
\mathrm{~h}_{2} \\
\vdots \\
\mathrm{h}_{\mathrm{L}}
\end{array}\right] \mathrm{x}+\left[\begin{array}{c}
\mathrm{n}_{1} \\
\mathrm{n}_{2} \\
\vdots \\
\mathrm{n}_{\mathrm{L}}
\end{array}\right]
$$

\section{2) Vector notation}

$$
\bar{y}=\bar{h} x+\bar{n}
$$

\section{3) Signal Detection}

If $\mathrm{y}_{1}, \mathrm{y}_{2}, \quad \mathrm{y}_{\mathrm{L}}$ are the received signals at the $\mathrm{L}$ antennas. Combining the received signals with complex weight conjugate gives us.

$$
r=w_{1}{ }^{*} y_{1}+w_{2}{ }^{*} y_{2}+\quad+w_{L}{ }^{*} y_{L}
$$

Where

$$
\begin{aligned}
& \text { Beam Former } \bar{w}=\left[\begin{array}{c}
w_{1} \\
w_{2} \\
\vdots \\
w_{L}
\end{array}\right] \\
& \bar{w}^{H}=\left[w_{1}{ }^{*}, w_{2}{ }^{*}, \quad w_{L}{ }^{*}\right]
\end{aligned}
$$

The received signal after the beam forming is

$$
\begin{aligned}
\bar{w}^{H} \bar{y} & \\
\bar{y} & =\bar{h} x+\bar{n} \\
\bar{w}^{H} \bar{y} & =\bar{w}^{H}(\bar{h} x+\bar{n}) \\
& =\bar{w}^{H} \bar{h} x+\bar{w}^{H} \bar{n}
\end{aligned}
$$

Where $\bar{w}^{H} \bar{h} x$ and $\bar{w}^{H} \bar{n}$ are the signal component and the noise component respectively of the received signal. The signal to noise ratio (SNR) of the received signal is the ratio of the signal power to the noise power.

$$
\begin{aligned}
& S N R=\frac{\text { Received Signal Power }}{\text { Noise Power }} \\
& \text { Received Signal }=\bar{w}^{H} \bar{h} x \\
& \text { Received signal power }=\left|\bar{w}^{H} \bar{h}\right|^{2} E\left\{|x|^{2}\right\} \\
& =\left|\bar{w}^{H} \bar{h}\right|^{2} P \\
& \text { Noise Power }=E\left\{\left|\bar{w}^{H} \bar{n}\right|^{2}\right\} \\
& =E\left\{\left(\bar{w}^{H} \bar{n}\right)\left(\bar{w}^{H} \bar{n}\right)^{*}\right\} \\
& =\sum\left|w_{i}\right|^{2} \sigma_{n}{ }^{2}
\end{aligned}
$$

Noise power at the output of the beam former $=\|\bar{w}\|^{2}{\sigma_{n}}^{2}$ or $\bar{w}^{H} \bar{w}{\sigma_{n}}^{2}$ 
Therefore SNR at the output of the beam former is given as:

$$
S N R=\frac{\left|\bar{w}^{H} \bar{h}\right|^{2} P}{\|\bar{w}\|^{2} \sigma_{n}{ }^{2}}
$$

The SNR can optimized by choosing $\bar{w}$ such that $\|\bar{w}\|^{2}=\bar{w}^{H} \bar{w}=1$

$$
S N R=\left|\bar{w}^{H} \bar{h}\right|^{2} \frac{P}{\sigma_{n}^{2}}
$$

In order to maximize

$$
\left|\bar{w}^{H} \bar{h}\right|^{2} \frac{P}{\sigma_{n}^{2}}
$$

But this is the dot product of two vectors

$$
\bar{a}^{H} \cdot \bar{b}=|\bar{a}||\bar{b}| \cos \theta
$$

This is maximize when $\cos \theta=1$ or $\theta=0$, which is to say that $\bar{a}$ is in the direction $\bar{b}$. Similarly, SNR is maximu $\mathrm{m}$ if $\bar{w}=c \bar{h}$ which means that $\|\bar{w}\|^{2}=c^{2}\|\bar{h}\|^{2}=1$

$$
c=\frac{1}{\|h\|}
$$

The optimal beam forming vector $\bar{w}$ that maximizes the received

$$
S N R=\frac{\bar{h}}{\|\bar{h}\|}
$$

The maximu m ratio combiner (MRC)

$$
\begin{aligned}
& \bar{w}_{\text {opt }}=\frac{\bar{h}}{\|\bar{h}\|} \\
& S N R=\mid \bar{w}^{H} \bar{h}^{2} \frac{P}{\sigma_{n}{ }^{2}} \\
& S N R=\|\bar{h}\|^{2} \frac{P}{\sigma_{n}{ }^{2}}
\end{aligned}
$$

d) Analysis of BER of Multiple Antenna System

where

$$
\begin{aligned}
\text { Received } S N R & =\|\bar{h}\|^{2} \frac{P}{\sigma_{n}{ }^{2}} \\
& =\left(\left|h_{1}\right|^{2}+\left|h_{2}\right|^{2}+\quad+\left|h_{L}\right|^{2}\right) \frac{P}{\sigma_{n}{ }^{2}}
\end{aligned}
$$

$$
g=\left|h_{1}\right|^{2}+\left|h_{2}\right|^{2}+\quad+\left|h_{L}\right|^{2}
$$

This gain $g=\|h\|^{2}$ is a Chi-square random variable with $2 \mathrm{~L}$ degrees of freedom. The distribution of Chi-square is given as:

$$
f_{G(g)}=\frac{1}{(L-1) !} g^{L-1} e^{-g}
$$




$$
\text { Received } S N R=g \frac{P}{\sigma_{n}^{2}}
$$

Hence the instantaneous bit error rate of multiple antenna wireless channels is given as:

$$
B E R=Q
$$

The average bit error rate can be obtained by taking the average of the distribution over the channel coefficient $f \quad G(g)$ as:

$$
B E R_{\text {Multiple Antenna }}=\int_{0}^{\infty} Q(\sqrt{g S N R}) f_{G(g)} d g
$$

The exact computation of the above expression for BER is quite complex, hence that is skipped here. The final expression for BER of this multiple antenna wireless with MRC is given as:

$$
B E R_{\text {Multiple Antenna }}=\left(\frac{1-\lambda}{2}\right)^{L} \sum_{l=0}^{L-1}{ }^{L+l-1} C_{l}\left(\frac{1+\lambda}{2}\right)^{l}
$$

Where

$$
\begin{gathered}
{ }^{n} C_{k}=\frac{n !}{k !(n-k) !} \\
\lambda=\sqrt{\frac{S N R}{2+S N R}}
\end{gathered}
$$

At high SNR

At high SNR

$$
\begin{aligned}
&\left(\frac{1-\lambda}{2}\right)=\frac{1}{2}\left(1-\sqrt{\frac{S N R}{2+S N R}}\right) \\
&=\frac{1}{2}\left(1-\frac{1}{\left(1+\frac{2}{S N R}\right)^{1 / 2}}\right) \\
& \cong \frac{1}{2}\left(1-\frac{1}{2}\left(\frac{2}{S N R}\right)\right) \\
& \cong \frac{1}{2 S N R}
\end{aligned}
$$

$$
\begin{aligned}
\frac{1}{2}(1+\lambda) & =\frac{1}{2}\left(1+\sqrt{\frac{S N R}{2+S N R}}\right) \\
& =\frac{1}{2}\left(1+\frac{1}{\left(1+\frac{2}{S N R}\right)^{1 / 2}}\right) \\
& \cong \frac{1}{2}\left(1+\left(1-\frac{1}{2} \frac{2}{S N R}\right)\right) \\
& \cong \frac{1}{2}\left(2-\frac{1}{S N R}\right) \\
& \cong \frac{1}{2} \cdot 2=1
\end{aligned}
$$




$$
\text { At high } S N R=\left\{\begin{array}{c}
(1-\lambda) / 2 \cong \frac{1}{2 S N R} \\
(1+\lambda) / 2 \cong 1
\end{array}\right.
$$

At high SNR the BER in multiple antenna wireless system can be simplified as:

$$
\begin{aligned}
B E R_{\text {Multiple Antenna }} & =\left(\frac{1-\lambda}{2}\right)^{L} \sum_{l=0}^{L-1}{ }^{L+l-1} C_{l}\left(\frac{1+\lambda}{2}\right)^{l} \\
& =\left(\frac{1}{2 S N R}\right)^{L-1} \sum_{l=0}^{L-1}{ }^{L+l-1} C_{l} \\
B E R_{\text {Multiple Antenna }} & ={ }^{2 L-1} C_{L} \frac{1}{2^{L}}\left(\frac{1}{S N R}\right)^{L}
\end{aligned}
$$

For our design consideration let take the BER of the LTE-A wireless communication system to be $10^{-6}$ [31]. In a single transmit antenna LTE-A system $\mathrm{L}=1$ where $\mathrm{L}$ represents the number of transmit antennas and $\mathrm{C}$ means combination [31].

$$
\begin{aligned}
& B E R_{\text {Single Antenna }} \\
& \begin{aligned}
{ }^{2 L-1} C_{L} \frac{1}{2^{L}}\left(\frac{1}{S N R}\right)^{L} \\
10^{-6}={ }^{2 \times 1-1} C_{1} \frac{1}{2^{1}}\left(\frac{1}{S N R}\right)^{1}=\frac{1}{2 S N R} \\
S N R=\frac{1}{2} 10^{6} \\
=500,000 \\
S N R(d B)=10 \log _{10} 500,000 \\
=56.99 \cong 57 \mathrm{~dB}
\end{aligned}
\end{aligned}
$$

In LTE-A system with two transmit antennas $\mathrm{L}=2$

$$
\begin{aligned}
& B E R_{\text {Multiple Antenna }}={ }^{2 L-1} C_{L} \frac{1}{2^{L}}\left(\frac{1}{S N R}\right)^{L} \\
& 10^{-6}={ }^{2 \times 2-1} C_{2} \frac{1}{2^{2}}\left(\frac{1}{S N R}\right)^{2} \\
& 10^{-6}={ }^{3} C_{2} \frac{1}{2^{2}}\left(\frac{1}{S N R}\right)^{2} \\
& 10^{-6}={ }^{3} C_{2} \frac{1}{2^{2}}\left(\frac{1}{S N R}\right)^{2}=\frac{3}{4} \frac{1}{S N R^{2}} \\
& S N R=\sqrt{750000} \\
& =866 \\
& S N R(\mathrm{~dB})=10 \log _{10} 866 \\
& =29.34 \mathrm{~dB}
\end{aligned}
$$

The amount of transmitted power saved between single antenna $\mathrm{L}=1$ and double antenna $\mathrm{L}=2$ in $\mathrm{dB}$ is given as:

$$
\begin{aligned}
& 10 \log _{10}\left(\frac{P_{1}}{P_{2}}\right)=57 d B-29.34 d B \\
& \frac{P_{1}}{P_{2}}=583 \\
& P_{1}=583 P_{2}
\end{aligned}
$$




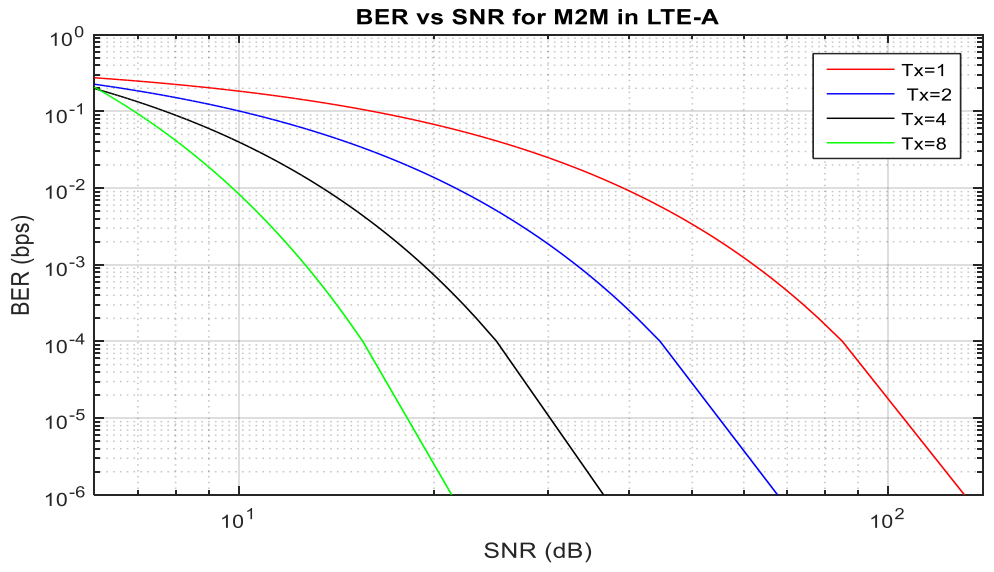

Figure 3. BER verses SNR in Rayleigh channel with multiple transmit antennas

For all intents and purposes the carrier frequency of LTE-A is 3.4-3.6 GHz, which means that the UE's can only have two transmit and receive antennas and the eNB can have up to 8 transit and receive antennas [31], [32]. In this regards we compared the power efficiency of LTE-A wireless communication in the downlink between one antenna and two antennas systems. The following outputs were obtained. In order to have a balanced comparis on between the microcell and the macrocell eNBs, let make average bit rate of all users as $30 \mathrm{Mb} / \mathrm{s} / \mathrm{Hz}$ [33]. The subscriber densities of urban and suburban areas can be assumed to be between 100-300 users $/ \mathrm{Km}^{2}$ [19]. The range of the microcell BS macrocell eNBs are $2 \mathrm{Km}$ and $35 \mathrm{Km}$ respectively [34].

\subsection{Power Efficiency}

The most significant factors in determining the power efficiency of the eNBs are the number of users served by the BS, the coverage area, the bandwidth and the power consumed.

$$
\text { Power Efficiency }=\frac{\text { Power consumed }}{\text { Bit Rate } \times \text { Number of users }}
$$

Number users $=$ Area $\times$ Subscriber Density
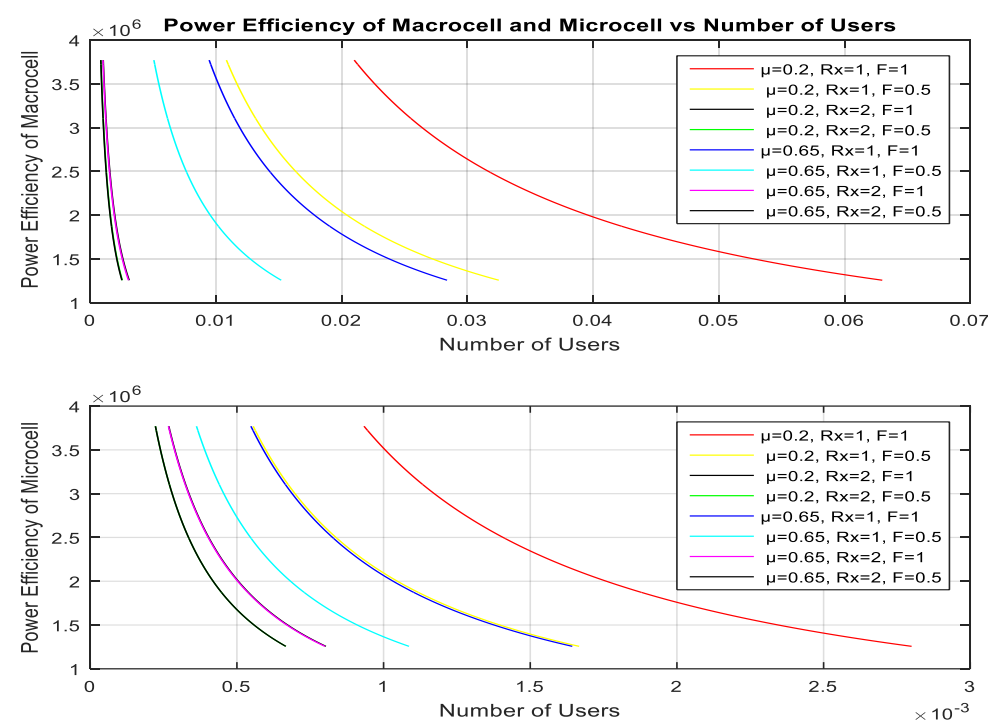

Figure 4. Power reduction strategies between macrocell and microcell eNBs 


\section{ANALYSIS OF SIMULATION}

It can be observed from the results obtained that for a macrocell eNBs operating at full load capacity $\mathrm{F}=1$ with the conventional amplifier along with a single antenna that the power consumed is very high, likewise the number active users served. This is depicted in the result obtained for macrocell eNB simulation first plot (red). Generally the macrocell eNBs are designed to be operated at their full capacity. If these macrocell eNBs are situated in the central business districts then during the weekdays between the hours of 8:00am to 5:00 pm the power consumed in serving these active users will be meaningful. However during the evenings and over the weekends when there is very little or no activity in these places, the network operator operating a macrocell eNB at such a place at full capacity risk expanding energy that will serve no meaningful purpose. For this reas on we propose a load factor of $\mathrm{F}=0.5$ be applied, which means that the macrocell eNBs operate at half of its rated capacity. In this way the power consumed will be considerably reduced as portrayed in the second plot of macrocell eNBs (yellow). Also if the macrocell eNB uses two transmitting antennas instead of one, here again the power consumed will be further reduced likewise the number of active users served as depicted in the simulation result obtained (black). Also if the mac rocell eNBs is made to use two transmitting antennas instead of one, here again the power consumed is further reduced, likewise the number of active users served as portrayed in the simulated result obtained (black). This means that by using two transmitting antennas at the macrocell eNBs, the power transmitted by each antenna will be considerably reduced. When the conventional amplifier in the macrocell eNBs along with two transmitting antennas and a load factor of $\mathrm{F}=0.5$ were implemented there was tremendous reduction in the power used and a corresponding reduction in the number of active users served as portrayed in the result obtained (green overlapping with black).

By introducing the Doherty power amplifier into the macrocell eNBs with an efficiency as high as 65 percent, the power used of the macrocell eNBs is very much reduced, even if the eNBs is operating at full installed capacity as depicted by the simulation result (blue). The result proved that with the Doherty power amplifier even when the macrocell eNBs is operating at full capacity the power consumed is less than that of a similar macrocell eNB operating at half of its capacity. This again points to the fact that much of the power consumed eNBs owing to the use of conventional amplifiers which are highly inefficient as portrayed by the result obtained in the simulation (blue). Now by applying a load factor of $F=0.5$ to the eNB, the power used is further reduced as depicted in the simulation result obtained (cyan). Again by employing two trans mitting antennas at the eNB and load factor $\mathrm{F}=1$ the power consumed is significantly reduced as portrayed in the simulation result (magenta). Here when the Doherty power amplifier is employed along with two transmitting antennas and a load factor of $F=0.5$ were applied the simulation result obtained coincided with the result obtained for the conventional amplifier with two transmitting antennas and a load factor of $\mathrm{F}=0.5$.

It can be observed from the simulation results obtained that when the conventional power amplifier was employed at full load capacity $\mathrm{F}=1$ in a single transmitting antenna system the power consumed was very high (red) likewise the number active users served. When a load factor of $F=0.5$ was applied to the microcell BS with the conventional amplifier, the power consumed was significantly reduced as portrayed in the simulation result obtained (yellow). It can be observed here that when two transmitting antennas were used along with the conventional amplifier at full load capacity $\mathrm{F}=1$ on the microcell BS, the power used was further reduced as portrayed in the result obtained from simulation (green overlapping with pink). When two antennas and a load factor of $\mathrm{F}=0.5$ were employed in the microcell $\mathrm{BS}$ with the conventional amplifier, the power used was very much reduced as portrayed in the simulation result obtained (black overlapping pink).

When the Doherty power amplifier was implemented in the microcell BS the results obtained from the simulation with one transmitting antenna and full load capacity $\mathrm{F}=1$ was an improvement on the result obtained for conventional amplifier at half load capacity (blue). Again when a load factor $\mathrm{F}=0.5$ was applied to the microcell BS with Doherty amplifier with one transmitting antenna there was further improvement in the power used as shown by the simulation result obtained (cyan) with a corresponding reduction in the number of active users served. By using two transmitting antennas in the microcell BS with Doherty amplifier along with load factor $\mathrm{F}=1$, the power used was again reduced as portrayed from the simulation result obtained (magenta) with a corres ponding reduction in the number active users served. Finally when the microcell BS with Doherty amplifier along with two transmitting antennas and a load factor $\mathrm{F}=0.5$ was implemented the result obtained from simulation coincided with the result obtained for microcell BS with the conventional amplifier along with two transmitting antennas and a load factor $\mathrm{F}=0.5$.

\section{CONCLUSION}

From the ensuing analysis it can be reasonably concluded that the high power consumption of the conventional LTE-A network can be reduced by incorporating multiple transmitting antennas at the eNBs 
and also by the use of more efficient power amplifiers such as the Doherty power amplifier. Again by introducing a load balancing technique into the operation of the eNBs the power consumption of the eNBs can significantly be reduced. However the power reduction comes along with a corresponding reduction in the number of active users that can be served per eNBs. When the power of the macrocell eNBs and microcell BS were reduced, the quality of service to the M2M devices and user equipments were maintained under various channel conditions. Again deploying microcell underneath the macrocell eNBs lead to a denser network but with less energy consumption which goes to improve the overall network power efficiency thereby improving the LTE-A network performance as a whole. This reduction in power consumption also leads to a reduction in the amount carbon emission into the atmosphere. Introducing more microcell BS into the LTE-A network will lead to frequent handovers when the macrocell eNBs are switched off. However we assume that most of the UE's or M2M devices are stationary devices such as vending machines, point of sale devices, utility meters, etc then the issue of hand over will not arise. Again injecting more microcell BS into the LTE-A network will reduce the distance between the BS and the M2M device and therefore the M2M devices will not have to expand so much energy in transmission.

\section{REFERENCE}

[1] Nokia Networks White Paper, "LTE-Advanced Evolution in Release 12-14 New Services to pave the way to 5G" ,www.resource.alcatel_lucent.com/asset/200175

[2] A.Ghosh, R. Ratasuk, B. Mondal, Nitin Mangalvedhe and T. Thomas, "LTE-advanced: next generation wireless broadband technology," IEEE Wireless Communications, Vol. 17, No. 3, pp. 10-22, June 2010.

[3] A. Khandekar, N. Bhushan, J. Tingfang and V. Vanghi, "LTE-advanced: heterogeneous networks," 2010 European Wireless Conference, 2010, pp. 978-982.

[4] Z. Hasan, H. Boostanimehr and V. K. Bhargava, "Green Cellular Networks: A Survey, Some Research Issues and Challenges", in IEEE Communications Survey and Tutorials, Vol. 13, No. 4, pp. 524-540, fourth Quarter 2011.

[5] K.M.S. Huq, S. Mumtaz, J. Bachmatiuk, J. Rodriguez, X. Wang, and R. L. Aguiar," in IEEE Transaction on Vehicular Technology, Vol. 64, No. 10, pp. 4670-4683, October 2015

[6] C. Desset et al., "Flexible power modelling of LTE base stations", 2012 IEEE Wireless Communications and Networking Conference, Shanghai, 2012, pp. 2858-2862.

[7] C. C. Hsu and J. M. Chang, "Spectrum-Energy Efficiency Optimization for Downlink LTE-A for Heterogeneous Networks," IEEE Transactions on Mobile Computing, Vol. 16 No. 5 pp. 1449-1461, May 12017.

[8] C. Y. Ho and C. Y. Huang, "Energy-Saving Massive Access Control and Resource Allocation Schemes for M2M Communications in OFDMA Cellular Networks," IEEE Wireless Communications Letters, Vol. 1, No. 3, pp. 209212, June 2012.

[9] N Abu Ali, "Enabling Technologies of Energy Efficient Cooperative M2M Networks: Benefits and Challenges," $38^{\text {th }}$ Annual IEEE Conference on Local Computer Networks-Workshops, Sydney, NSW, 2013, pp. 987-993.

[10] D. Sabella et al, "Energy Efficiency Benefits of RAN-as-a-Service Concept for a Cloud-Based 5G Mobile Network Infrastructure," IEEE Access, 2014, Vol. 2, No. pp. 1586-1597, 2014.

[11] M. Behjati, R. Nordin and M. Ismail, "Compensation of Energy-Efficiency Degradation Due to Antenna-Muting in Distributed Antenna Systems for a Green Cellular Network," 2015 IEEE $11^{\text {th }}$ international Conference on Wireless and Mobile Computing, Networking and Communications (WiMob), Abu Dhabi, 2015, pp 336-340.

[12] B. François and P. Reynaert, "Highly Linear Fully Integrated Wideband RF PA for LTE-Advanced in 180-nm SOI," IEEE Transactions on Microwave Theory and Techniques, Vol. 63, No.2, pp. 649-658, February 2015.

[13] Y. Sun and X. Zhu, "Broadband Continuous Class- $\mathrm{F}^{1}$ Amplifier with Modified Harmonic-Controlled Network for Advanced Long Term Evolution Application," in IEEE Microwave and Wireless Components Letters, 2015, Vol. 25, No. 4, pp. 250-252, April 2015.

[14] X. W. Zhu et al., "Doherty Power Amplifiers with Improved Load Modulation for $100 \mathrm{MHz}$ LTE-advanced Application," Proceedings of 2014 3rd Asia-Pacific Conference on Antennas and Propagation, Harbin, 2014, pp. 1303-1305.

[15] K. Hiltunen, "Utilizing eNodeB sleep mode to improve the energy -efficiency of dense LTE networks," 2013 IEEE 24th Annual International Symposium on Personal, Indoor, and Mobile Radio Communications (PIMRC), London, 2013, pp. 3249-3253.

[16] M. Lakshmanan, V. N. Mohammed, M. Palanivelan and P. Siva Kumar, "Energy Control in 4 G Networks," Indian Journal of Science and Technology (IJST), Vol. 9, No. 35, September 2016.

[17] B. Shah, N. Thakker, G. Dalwadi and N. Koyhari, "Modified Output Combining 3-Stage Doherty Power Amplifier Design for LTE-Micro eNodeB," International Journal of Wireless Microwave Technologies (IJWMT), Vol. 5, No. 5, pp. 10-24, September 2015.

[18] C. Fan, X. W. Zhu J. Xia and L. Zhang, "Efficiency enhanced class-F Doherty power amplifier at $3.5 \mathrm{GHz}$ for LTE-Advanced applications," 2013 Asia-Pacific Microwave Conference Proceedings (APMC), Seoul, 2013, pp. 707-709.

[19] M. Deruyck et al., "Reducing the power consumption in wireless access networks: overview and recommendations", (2012) Progress in Electromagnetics Research-PIER. 132, pp. 255-314, 2012. 
[20] M. Deruyck, E. Tanghe, W. Joseph and L. Martens, "Modelling the energy efficiency of microcell base stations," In 1st International Conference on Smart Grids, Green Communications and IT Energy-Aware Technologies (ENERGY-2011) pp. 1-6, IARIA, 2011.

[21] A. Chatzipapas, S. Alouf, and V. Mancuso, "On the Minimization of Power Consumption in Base Stations using on/off Power Amplifiers," 2011, IEEE Online Conference on Green Communications, New York, 2011, pp. 18-23.

[22] M. Toufique, H. Jiang and A. R. Hadi, "Duty cycling stratagems for network permanency in wireless sensor network", in Indonesian Journal of Electrical Engineering and Computer Science (IJEECS), Vol. 3, No. 3, Dept., 2016, pp. 534-539

[23] M. Deruyck, W. Joseph, B. Lannoo, D. Colle, and L. Martens, "Designing Energy-Efficient Wireless Access Networks: LTE and LTE-Advanced," in IEEE Internet Computing, Vol. 17, No. 5, pp. 39-45, Sept.-Oct. 2013.

[24] M. Dottling and I. Viering, "Challenges in Mobile Network Operation: Towards Self-Optimizing Networks," 2009 IEEE International Conference on Acoustics, Speech and Signal Processing, Taipei, 2009, pp. 3609-3612.

[25] 4 G Americas White Paper ,2011, Self-Optimizing Networks: The Benefits of SON in LTE

[26] ETSI TR 136902 V9.2.0 (2010-09), "LTE; Evolved Universal Terrestrial Radio Access Network (E-UTRAN); Self-configuring and self-optimizing network (SON) use cases and solutions (3GPP TR 36.902 version 9.2.0 Release 9)

[27] Nokia networks white paper, "LTE-A Optimizing LTE for the Internet of Things White Paper", https://novotech.com/docs/.../LTE-A-optimizing-lte-for-the-internet-of-things.pdf?

[28] Nokia White Paper, "LTE Evolution for IoT Connectivity White Paper", resources.alcatel-lucent.com/asset/200178

[29] A.A. Amin, M.S. Islam, A.A.M. Masud and M.N.H. Khan, "Design and performance analy sis of $1.8 \mathrm{Ghz}$ low noise amplifier for wireless receiver applications," in Indonesian Journal of Electrical Engineering and Computer Science (IJEECS), Vol. 6, No. 3, pp. 656-662, June 2017.

[30] J. Xia, X. Zhu, L. Zhang, J. Zhai and Y. Sun, "High-Efficiency GaN Doherty Power Amplifier for 100-MHz LTEAdvanced Application Based on Modified Load Modulation Network," in IEEE Transaction on Microwave Theory and Techniques, 2013, Vol. 63, No. 8, pp. 2911-2921, August 2013.

[31] A. K. Jagannatham, "Principles of Modern Wireless Communication Systems: Theory and Practice", McGraw Hill Education (India) Private Limited, New Delhi, pp. 60-63

[32] X. Zhang, Y. Zhang, R. Yu, W. Wang and M. Guizani, "Enhancing Spectral Efficiency for LTE-Advanced Heterogeneous Networks: Users Social Pattern Perspective," in IEEE Wireless Communications, Vol. 21, No. 2, pp. 10-17 April 2014.

[33] W. Vereecken et al., "Power Consumption in Telecommunication Networks: Overview and Reduction Strategies" in IEEE Communications Magazine, Vol. 49, No. 6, pp. 62-69, June 2011.

[34] A. Zabri, M.K.A. Rahim, F. Zubir, N.M. Nadzir, and H.A. Majid, "Video monitoring application using wireless sensor nodes with various external antenna," in Indonesian Journal of Electrical Engineering and Computer Science (IJEECS), Vol. 6, No. 1, pp. 148-154, April 2017. 